\title{
Color-based inhibition of return
}

\author{
MARK B. LAW, JAY PRATT, and RICHARD A. ABRAMS \\ Washington University, St. Louis, Missouri
}

\begin{abstract}
The inhibition of return of visual attention based on stimulus color was examined in three experiments. In the first experiment, a discrete trial paradigm showed that subjects were slower to detect a color patch if the color matched that of a patch presented earlier in the same location. Experiment 2 showed that the inhibition only occurs if a neutral, nontarget distractor color is presented between presentation of the initial color cue and the subsequent target patch-explaining the previous failures to detect the phenomenon. Experiment 3 extended the results to a different combination of colors. Taken together, the results have important implications for the processing of stimulus attributes such as spatial location and color, and for the conditions necessary for producing inhibition of return.
\end{abstract}

People are slower in directing their attention to a recently attended location than to a location that they have not previously attended. This phenomenon, termed $i n h i-$ bition of return (Posner \& Cohen, 1984), is believed to enhance the efficiency of visual search by creating a bias toward sampling novel locations (Posner \& Cohen, 1984; Tipper, Weaver, Jerreat, \& Burak, 1994). Inhibition of return affects both manual responses (e.g., Posner $\&$ Cohen, 1984; Maylor \& Hockey, 1985) and eye movements (e.g., Abrams \& Dobkin, 1994; Vaughan, 1984). To date, inhibition of return has been demonstrated only for spatial attributes of a stimulus. However, if the inhibition is a result of a mechanism that enhances the sampling of new information, it might be expected that inhibition would also affect nonspatial attributes of stimuli, such as color. The aim of the present research was to investigate inhibition of return based on stimulus color.

\section{Object-Based Inhibition}

Several researchers have reported that inhibition of return occurs for particular objects, not just for the location at which an object has been attended (e.g., Abrams \& Dobkin, 1994; Tipper, Driver, \& Weaver, 1991; Tipper et al., 1994). That is, people are slower to return attention to a previously attended object even if the object has moved since it was first attended. Such object-based inhibition requires some way for the mechanisms that produce inhibition to distinguish the previously attended object from other objects in the environment (Tipper et al., 1991; Tipper et al., 1994). One way that this could be accomplished is for inhibition to be associated with attended attributes that may enable one to discriminate between objects, such as color or shape. Determining

This research was supported by Grant R29-MH45145 from the National Institutes of Health to R.A.A. We thank Heidi Korthase for her excellent work in collecting the data. Correspondence concerning this article should be sent to M. B. Law, Department of Psychology, Campus Box 1125, Washington University, St. Louis, MO 63130 (e-mail: mlaw@artsci.wustl.edu). whether inhibition of return exists for attributes such as these may also provide insight into the relationship between spatial and nonspatial attributes. It may indeed be that inhibition of return exists only for spatial location because spatial location is a special stimulus attribute (Kwak \& Egeth, 1992).

\section{Color-Based Inhibition}

Kwak and Egeth (1992) performed a series of experiments intended to detect inhibition of return based on spatial and nonspatial stimulus attributes. They found a reliable 5-msec inhibition effect based on stimulus color in one experiment, and nonsignificant trends consistent with color-based inhibition in two other experiments. But the significant effect did not reappear under conditions that partially replicated the earlier experiment. Also, color-based inhibition was not present in a somewhat different condition that was designed to increase the subjects' need to attend to the stimulus color. Two additional experiments failed to reveal inhibition of return based on another nonspatial attribute (line orientation), although strong space-based inhibition of return was observed in all experiments. Thus, Kwak and Egeth concluded that spatial location was special because inhibition of return operates only for location and not for other stimulus attributes. Such a conclusion is consistent with a number of other formulations that have also assumed a special status for spatial location (e.g., Tsal \& Lavie, 1988, 1993). But there is reason to believe that Kwak and Egeth's (1992) experiments did not provide a strong test of the possible existence of color-based inhibition of return. This possibility arises as a result of details of the method that they used in their experiments.

Kwak and Egeth (1992) used a continuous responding paradigm in which the stimulus on a given trial served as the attentional precue for the next trial. Such a paradigm has been previously used to examine inhibition of return to spatial locations (e.g., Maylor \& Hockey, 1985, 1987). However, although the continuous responding paradigm may be sufficient to reveal spatially based in- 
hibition, it may not be appropriate for detection of other types of inhibition. The reason for this hinges on a key aspect of the procedure - and on the definition of inhibition of return itself. Specifically, in order to demonstrate inhibition of return, it is necessary to (1) direct attention to a value of a specific stimulus dimension (e.g., a particular spatial location or color), and (2) then remove attention from that particular value of the cued stimulus dimension. Only after attention has first been allocated, and then removed, does the possibility exist for inhibition of return. This is because the inhibition is in returning attention to a previously attended, but then unattended, value of the relevant stimulus dimension. We argue (and will present evidence below) that the continuous responding paradigm may indeed fulfill both prerequisites when space is the relevant stimulus dimension, but that it fails to provide the second prerequisite (viz., removal of attention) when color is the dimension under consideration. If this is true, the paradigm simply cannot be used to make inferences about the existence of color-based inhibition.

In the continuous responding paradigm used by Maylor and Hockey $(1985,1987)$ and Kwak and Egeth (1992), subjects fixate a central point while stimuli are presented peripherally, one after the other, with a short time interval between them. The subjects are to respond to each stimulus with a keypress. The latency to detect a given stimulus is reported as a function of the relation between the stimulus and the most recently presented earlier one. A typical finding is that subjects are slower to detect a stimulus if it appears at the same location as the previous one (e.g., Kwak \& Egeth, 1992). A key aspect of this continuous responding procedure is that no stimulus is presented expressly for the purpose of removing the subject's attention from the previous stimulus. Instead, it is presumed that subjects naturally return their attention to the central fixation location during the interval between one stimulus and the next. Subjects are motivated to do this, because the array of potential stimulus locations is usually centered at fixation, and the locations are equiprobable. Indeed, Posner and Cohen (1984) presented evidence that subjects will naturally return their attention to fixation in such a situation; there is no need for an explicit cue. This aspect of the procedure makes the continuous responding paradigm efficient for the study of spatially based inhibition of return, yet this feature also renders it inappropriate for inhibition of return based on other stimulus attributes. A nontarget spatial location is always available for attention to return to naturally; the spatial locations simply exist and are always accessible. But a nontarget color is not available unless it is explicitly presented. Thus, in the interval between one stimulus and the next, subjects might naturally be expected to remove their attention from the spatial location of the previous target-but not necessarily from its color or other attributes. ${ }^{1}$ The continuous responding paradigm may not create the conditions necessary for examining inhibition of return to nonspatial stimulus attributes. If this is true, it may be premature to conclude that inhibition of return does not operate for color.

\section{Overview of Experiments}

Our goal was to reexamine inhibition of return to stimulus color by using a discrete-trial paradigm that would not suffer from the limitations of the continuous responding paradigm described above. In the first experiment, we show that color-based inhibition can be obtained when a cue is presented that causes subjects to remove their attention from the color of the first stimulus. In the second experiment, we show that the inhibition disappears if the neutral stimulus is omitted. In the third experiment, we extend the results to a different combination of stimulus colors.

\section{EXPERIMENT 1}

The goal of our first experiment was to use a discretetrial paradigm to investigate inhibition of return to color. In this paradigm, each trial is presented in isolation, and attention is explicitly summoned first to the initial stimulus color and then away from it. This solves the potential problem associated with removing attention from the initially cued stimulus (or the lack thereof) which exists in the continuous responding paradigm. In the present experiment, two colored stimuli (each either red or blue) were presented successively at the same location, and subjects made a speeded response to the onset of the second stimulus. To divert attention to a neutral (i.e., nontarget) color, a different colored patch (magenta) was always presented between the two stimuli. If inhibition of return occurs for stimuli of the same color, responses should be slower when both stimuli are the same color (e.g., both red) than when they are different colors (e.g., red and blue).

\section{Method}

Subjects. Twenty-six Washington University undergraduates were each paid $\$ 5$ and served in a single 30 -min session. All had normal or corrected-to-normal vision.

Apparatus and Procedure. The subjects were tested individually in a dimly lit room where a microcomputer with a color monitor was used to present stimuli and record responses. The stimuli were magenta, red, and blue squares, presented at a viewing distance of $40 \mathrm{~cm}$ and subtending . $6^{\circ}$ of visual angle on each side. All stimuli were presented at a central fixation location against a uniform gray background.

The sequence of events on a trial is shown in Figure 1. Each trial began with a 150 -msec warning tone followed by presentation of a magenta square which served as a fixation target and remained on the screen for $510 \mathrm{msec}$. The magenta square was replaced by either a red or a blue square, the cue, which was presented for $900 \mathrm{msec}$. A magenta square, the neutral attractor, always followed the cue and was also presented for $900 \mathrm{msec}$. We call this the neutral attractor because the square was neutral with respect to the color of the subsequent target, and at the same time, it was designed to attract the subject's attention away from the color of the cue. Next, either a red or blue square, the target, was presented on noncatch trials. Subjects were instructed to respond as quickly as possible to the onset of the target by pressing the space bar on the computer keyboard with the index finger of their dominant hand. On catch trials, no target was presented and only the gray 
background was visible. Subjects were instructed not to respond on the catch trials, (i.e., when no target was presented). During the $1,000-\mathrm{msec}$ intertrial interval that followed each response, only the gray background was visible. Reaction time was measured from the onset of the target square until the space bar was pressed. An error tone was sounded and an error message was presented on the screen if a response was made before target onset, if no response was produced within $1,000 \mathrm{msec}$ after target onset, or if a response was made on a catch trial.

There were two types of trials with targets: repeated and nonrepeated. Repeated trials had cue and target squares of the same color, either both red or both blue. Nonrepeated trials had cue and target squares of different colors, one red and the other blue.

Design. Each subject served in a total of 250 trials; 100 were repeated trials, 100 were nonrepeated trials, and 50 were catch trials. Half of the repeated trials and half of the nonrepeated trials had red targets; the other half had blue targets. Trials from the various conditions were presented in random order, with the constraint that no more than 3 consecutive trials were of the same type or had the same color targets. Trials were divided into 10 blocks of 25 . At the end of each block, subjects were shown the mean reaction time for all correct responses during that block and they were allowed a short break.

\section{Results}

Trials on which an error occurred or with a reaction time of less than $100 \mathrm{msec}$ or greater than $500 \mathrm{msec}$ were not included in the analysis. These constituted less than $5 \%$ of the total and did not differ between conditions.

Reaction times are shown in Figure 2. As can be seen, there was a $5.5-\mathrm{msec}$ inhibition of return effect: Repeated trials $(284.0 \mathrm{msec})$ were slower than nonrepeated trials $(278.5 \mathrm{msec})[F(1,25)=11.7, p<.005]$. Twenty of the 26 subjects showed this effect.

Subjects were also $7.5 \mathrm{msec}$ faster to respond to blue targets than to red targets $(277.5$ and $285.0 \mathrm{msec}$, respectively) $[F(1,25)=22.2, p<.0005]$. However, target color did not interact with color repetition (i.e., repeated vs. nonrepeated) $[F(1,25)<1]$.

\section{Discussion}

The results of Experiment 1 clearly establish the existence of color-based inhibition of return. The subjects were slower to detect the target when they had previ-

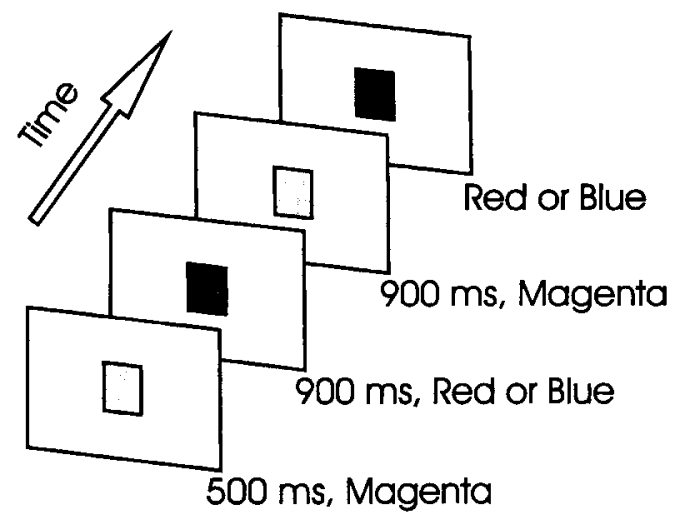

Figure 1. Example of the events that occurred during a trial of Experiment 1. The duration (in milliseconds) and color of each display are indicated beside each frame.

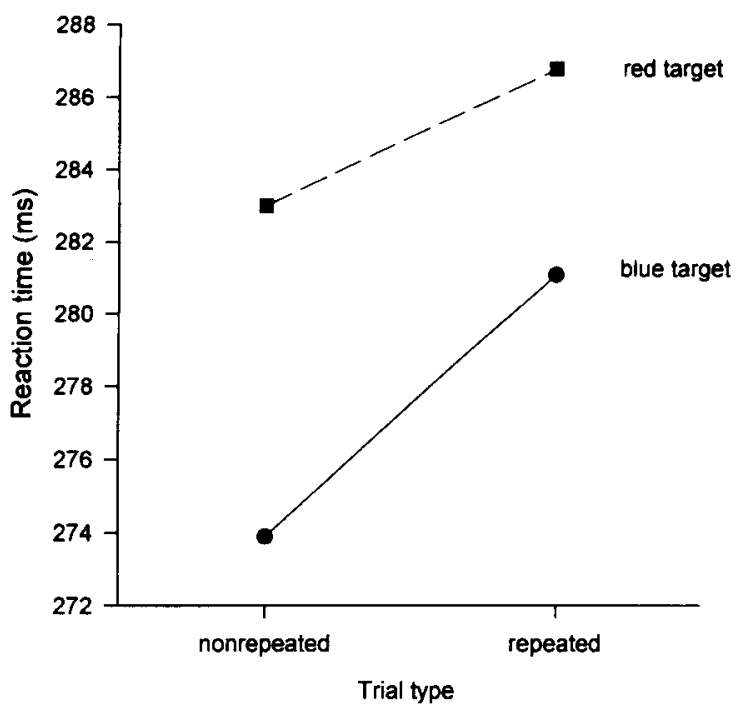

Figure 2. Mean reaction time in Experiment 1 for responses to the target square as a function of trial type and target color.

ously attended but then removed attention from the same color: red targets were more difficult to detect when the cue had been a red square (as opposed to a blue one), and subjects were slower to detect the blue target when the cue had been blue. Presumably, we were able to demonstrate color-based inhibition because the neutral attractor served to draw the subject's attention away from the cue color; in earlier unsuccessful attempts, a neutral attractor had not been used (see, e.g., Kwak \& Egeth, 1992).

\section{EXPERIMENT 2}

Having demonstrated color inhibition of return, we sought to show that the reason why we were able to find inhibition of return to color was that we included a neutral, nontarget stimulus between cues and targets (i.e., the magenta square). The present experiment was therefore identical to Experiment 1 in all ways save one: the exclusion of the nontarget magenta square. If it is necessary to have a nontarget stimulus to divert attention from target stimuli, removal of the magenta square should eliminate inhibition of return to color.

\section{Method}

Subjects. Twenty-six Washington University undergraduates who did not participate in Experiment 1 were each paid \$5 and served in a single 30-min session. All had normal or corrected-tonormal vision.

Apparatus and Procedure. This experiment was identical to that of Experiment 1 with the exception that there was no neutral attractor or fixation square. Only the gray background was visible during the times when the neutral attractor or fixation square would have been displayed in Experiment 1.

\section{Results}

Errors accounted for less than 3\% of the trials and did not differ between conditions. As is shown in Figure 3, 
the mean reaction times for repeated and nonrepeated trials were 258.1 and $259.5 \mathrm{msec}$, respectively, and were not reliably different $[F(1,25)=2.7, p>.1]$. There was also no reliable difference between mean RTs for red and blue targets, which were 259.0 and $258.7 \mathrm{msec}$, respectively $[F(1,25)<1]$. Also, trial type and target color did not interact $[F(1,25)<1]$.

Another analysis was performed, with data from Experiments 1 and 2 forming one level of an additional between-groups factor. There was an overall difference in response time between repeated $(271-\mathrm{msec})$ and nonrepeated $(269-\mathrm{msec})$ trials $[F(1,50)=5.1, p<.05]$. The effect of trial type interacted with experiment, showing that the difference between repeated and nonrepeated trials was reliably greater in Experiment $1(5.5 \mathrm{msec})$ than in Experiment $2(-1.4 \mathrm{msec})[F(1,50)=14.4, p<.001]$. Thus, the inhibition demonstrated in Experiment 1 was not present in Experiment 2. Reaction times were also somewhat faster overall in Experiment 2 than in Experiment 1 (259 and $281 \mathrm{msec}$, respectively) $[F(1,50)=9.1$, $p<.005]$. Blue targets $(268 \mathrm{msec})$ also were responded to more quickly than red targets $(272 \mathrm{msec})[F(1,50)=$ $13.7, p<.001]$. The difference in response times for blue and red targets was larger in Experiment $1(7.5 \mathrm{msec})$ than in Experiment $2(0.3 \mathrm{msec})[F(1,50)=12.1, p<$ $.005]$. However, it is important that there was no interaction between trial type and target color $[F(1,50)<1]$, indicating that the inhibition did not depend on target color, but rather on whether a trial was repeated or nonrepeated. Also, in support of this, there was no interaction between trial type, target color, and experiment $[F(1,50)<1]$.

\section{Discussion}

Experiment 2 was identical to Experiment 1, with the exception that the neutral attractor (i.e., the magenta

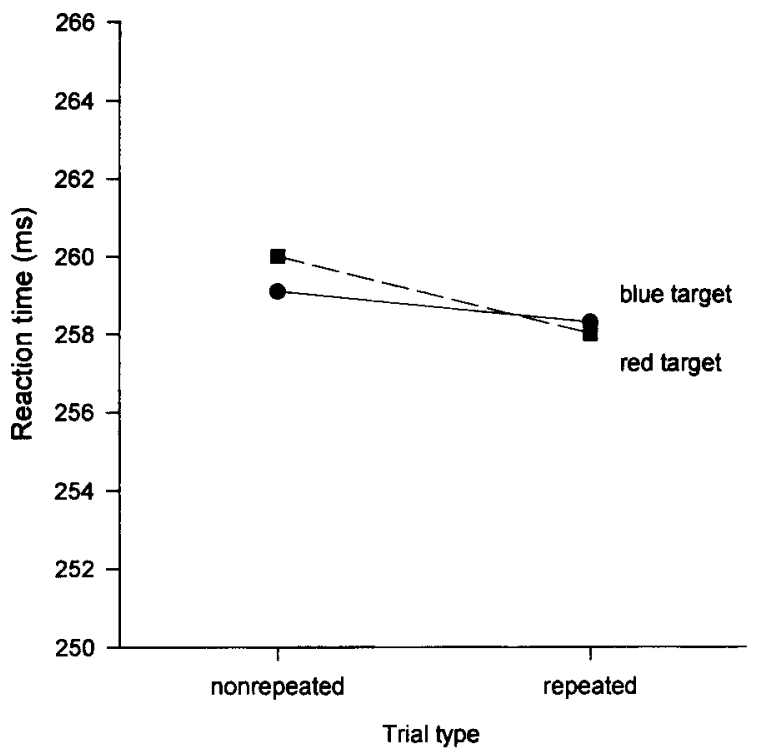

Figure 3. Mean reaction time in Experiment 2 for responses to the target square as a function of trial type and target color. square presented between the cue and target squares) was not present in Experiment 2. Instead, only the gray background was visible. The result of this change was that the inhibition that was present in Experiment 1 was not present in Experiment 2. This finding supports the assertion that to produce inhibition of return, it is necessary to cause attention to be removed from one value of an attended stimulus attribute and be directed toward a different value of the same attribute (i.e., from a target location to a neutral location, or from a target color to a neutral color). The present results also argue against the possibility that subjects in Experiment 1 were slower to respond to repeated color stimuli because of chromatic adaptation to the repeated color. If that were the case, inhibition would be expected to have occurred in both experiments. We address this possibility further in the Appendix, where we report an experiment in which subjects' thresholds were measured after red and blue cues. The results show that chromatic adaptation is not a viable explanation of the present results.

Reaction times also were shorter in Experiment 2 than in Experiment 1. This may have occurred because the color change that signaled the appearance of the target in Experiment 1 may have been a less salient event than the abrupt onset that signaled the appearance of the target in Experiment 2 (see Theeuwes, 1994).

\section{EXPERIMENT 3}

It is possible that there was something special about the relationship between the neutral attractor color and the target colors that was responsible for the inhibition of return found in Experiment 1. This seems possible, given that a difference was found between response times to red and to blue targets in Experiment 1 but not in Experiment 2. Perhaps responses to red targets in Experiment 1 were slower because red targets were more difficult to distinguish from the magenta neutral attractor than were blue targets. Such an occurrence, if true, does not necessarily compromise our conclusions about color-based inhibition of return. However, it does leave open the possibility that some idiosyncratic feature of the stimuli used in Experiment 1 was responsible for the observed effect. To rule out this possibility, Experiment 3 was designed to provide a replication of Experiment 1 with green rather than magenta as the neutral attractor color. Otherwise, Experiment 3 and Experiment 1 were identical.

\section{Method}

Subjects. Ten Washington University undergraduates who did not participate in either Experiment 1 or Experiment 2 were each paid $\$ 5$ and served in a single 30-min session. All had normal or corrected-to-normal vision.

Apparatus and Procedure. This experiment was identical to Experiment 1, with the exception that the color of the neutral attractor and the fixation square was green here, instead of magenta as it had been previously.

\section{Results}

Errors constituted less than $5 \%$ of the trials and did not differ between conditions. There was a $6.2-\mathrm{msec}$ inhi- 


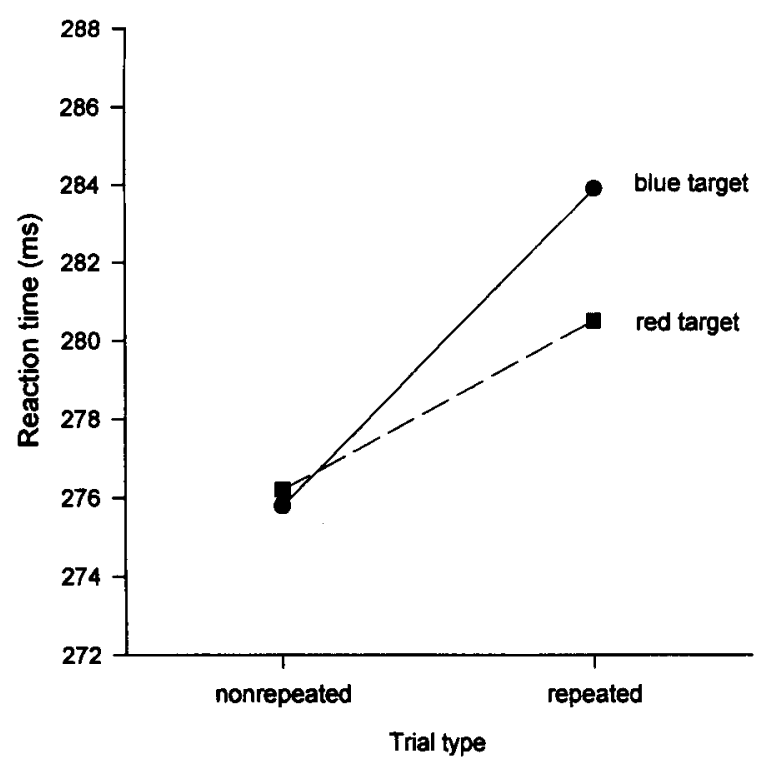

Figure 4. Mean reaction time in Experiment 3 for responses to the target square as a function of trial type and target color.

bition effect. As can be seen in Figure 4, repeated trials $(282.2 \mathrm{msec})$ were slower than nonrepeated trials $(276.0 \mathrm{msec})[F(1,9)=6.8, p<.05]$. Eight of the 10 subjects showed this effect. There was no effect of target color (blue, $279.8 \mathrm{msec}$; red, $278.3 \mathrm{msec}$ ) $[F(1,9)<1]$, nor did target color interact with the type of trial $[F(1,9)=3.3, p>.05] .^{2}$

\section{Discussion}

Experiment 3 was identical to Experiment 1 except that the neutral attractor and fixation square were green instead of magenta. Nevertheless, we replicated the color-based inhibition of return result of Experiment 1. In addition, red and blue targets were apparently equally distinguishable from the green neutral attractor, as is evidenced by the absence of a main effect of target color. Thus, the inhibition of return found in Experiment 1 was not due to some special combination of neutral attractor and stimulus colors used in that experiment, since the effect was obtained here with a differently colored neutral attractor.

\section{GENERAL DISCUSSION}

In the present paper, we have reported results which establish the existence of color-based inhibition of return. In Experiment 1, subjects were slower to detect a color patch if a cue of the same color had been previously attended but then unattended. In Experiment 2, we showed that the inhibition was not present when subjects were not provided with a color patch that could remove their attention from the earlier cued color. In Experiment 3, we replicated and extended the results of Experiment 1 by using a different color neutral patch.

\section{Relation to Previous Research on Color-Based Inhibition}

In addition to establishing the presence of inhibition of return based on stimulus color, the present experiments provide an explanation for the inability of previous researchers to reliably demonstrate such inhibition. In particular, the continuous responding paradigm used previously (e.g., Kwak \& Egeth, 1992) may not provide an opportunity for subjects to remove their attention from the initially cued color - a condition which may be necessary in order to produce inhibition of return. Such a limitation has not affected uses of the procedure to examine inhibition of return in the spatial domain. In the spatial domain, many nontarget locations are continuously available to which attention may be directed after the initial cue. Indeed, subjects are motivated to redirect their attention to the center of the stimulus array if time permits, because the location of the cue typically does not predict the location of the subsequent target (Posner \& Cohen, 1984). However, for a nonspatial attribute such as color, alternative nontarget colors are not typically available to attract the subject's attention. Furthermore, there does not appear to be any reason for the subject to attempt to direct his/her attention to a nontarget color. Instead, if attention is to be removed from the cued color, a nontarget color (what we call the neutral attractor) must be explicitly provided.

\section{Implications for Inhibitory Mechanisms}

The present results also have implications for inhibitory mechanisms more generally. In particular, although spatial location is a very important stimulus attribute, it does not appear to be as special as has previously been believed. In particular, space is not the only dimension for which one might be inhibited in the return of attention. As suggested earlier, if inhibition of return is object centered, the inhibition might be expected to be sensitive to attributes that define objects, even when they move. Such attributes include color, among others.

\section{Relation to Repetition Blindness}

An interesting similarity exists between our findings and those of Kanwisher (1991) regarding repetition blindness. Repetition blindness is a relative inability to detect or recall repetitions of items that occur in a rapid serial visual presentation (Kanwisher, 1987, 1991). Kanwisher (1991) presented sequences of colored symbols, with a pair of same-colored stimuli separated by a differently colored stimulus. She found repetition blindness for the second occurrence of the repeated color stimulus. No symbols were repeated in these sequences, indicating that the repetition blindness effect was color based. At first, the similarity of Kanwisher's (1991) paradigm and results to those in the present study suggests that a common mechanism may underlie both effects. However, the interval between the repeated color stimuli used by Kanwisher (1991) was $117 \mathrm{msec}$, whereas intervals of at least $300 \mathrm{msec}$ typically produce inhibition of 
return in covert orienting of attention paradigms (Posner \& Cohen, 1984). In fact, intervals of less than $300 \mathrm{msec}$ usually produce facilitation in such paradigms. Also, repetition blindness effects have been found for repeated semantic concepts (MacKay \& Miller, 1994) and repeated auditorily presented words (Miller \& MacKay, 1994), further suggesting that repetition blindness and inhibition of return are subserved by different processing mechanisms.

\section{Relation to Other Work}

The finding of color-based inhibition of return in the present study is also consistent with previous research on selective attention and color. First, the finding of inhibition of return for color indicates that color may be attended selectively. This is consistent with Treisman's feature integration theory (Treisman, 1988; Treisman \& Gelade, 1980), which suggests that color is processed both separately and in parallel with other featural attributes. Additionally, Hillyard and Münte (1984) and Humphreys (1981) found evidence that stimulus color can be attended to independently of stimulus location. Second, the color-based inhibition effect (approximately $5-6 \mathrm{msec}$ ) is considerably smaller than the inhibition of return effect found for spatial locations (typically 20 $30 \mathrm{msec}$ ). This finding is consistent with previous findings that spatial information has precedence over color information (Hillyard \& Münte, 1984; Tsal \& Lavie, 1988,1993 ), although color information could interact with location information (Lambert \& Corban, 1992). Finally, we studied color-based inhibition of return with stimuli that always appeared at the same spatial location (i.e., there was no spatial uncertainty). As Tsal and Lavie $(1988,1993)$ have shown, nonspatial attributes of a stimulus (such as color) are more likely to be attended if attention is directed to the location of the stimulus. Thus, the color-based inhibition of return that we observed might be reduced or eliminated in situations with spatial uncertainty.

\section{Conclusion}

The results of this study show that inhibition of return can occur for nonspatial stimulus attributes. Such inhibition might underlie object-centered inhibition of return because attributes like color might distinguish one object from another. This would be especially useful if the objects were in motion. The results reported here underscore the need for the further examination of inhibition of return for attributes other than spatial location.

\section{REFERENCES}

Abrams, R. A., \& Dobkin, R. S. (1994). Inhibition of return: Effects of attentional cuing on eye movement latencies. Journal of Experimental Psychology: Human Perception \& Performance, 20, $467-$ 477.

HillyaRd, S. A., \& MÜNTE, T. F. (1984). Selective attention to color and location: An analysis with event-related brain potentials. Perception \& Psychophysics, 36, 185-198.
HumphreYs, G. W. (1981). Flexibility of attention between stimulus dimensions. Perception \& Psychophysics, 30, 291-302.

KANWISHER, N. G. (1987). Repetition blindness: Type recognition without token individuation. Cognition, 27, 117-143.

KANWISHER, N. G. (1991). Repetition blindness and illusory conjunctions: Errors in binding visual types with visual tokens. Journal of Experimental Psychology: Human Perception \& Performance, 17, 404-421.

KWAK, H.-W., \& EGETH, H. (1992). Consequences of allocating attention to locations and to other attributes. Perception \& Psychophysics, $51,455-464$

LAmbert, A. J., \& Corban, R. (1992). Spatial attention and expectancy for colour, category and location: Further evidence against the spotlight model. Acta Psychologica, 81, 39-51.

MacKay, D. G., \& Miller, M. (1994). Semantic blindness: Repeated concepts are difficult to encode and recall under time pressure, $P s y$ chological Science, 5(1), 52-55.

MAYLOR, E. A., \& HoCKEY, R. (1985). Inhibitory component of externally controlled covert orienting in visual space. Journal of Experimental Psychology: Human Perception \& Performance, 11, 777 787.

MAYLOR, E. A., \& HoCKEY, R. (1987). Effects of repetition on the facilitatory and inhibitory components of orienting in visual space. Neuropsychologia, 25, 41-54.

MilleR, M., \& MACKAY, D. G. (1994). Repetition deafness: Repeated words in computer-compressed speech are difficult to encode and recall. Psychological Science, 5(1), 47-51.

POSNER, M., \& COHEN, Y. (1984). Components of visual orienting. In H. Bouma \& D. G. Bouwhuis (Eds.), Attention and performance $X$ (pp. 531-556). Hillsdale, NJ: Erlbaum.

THEEUWES, J. (1994). Stimulus-driven capture and attentional set: Selective search for color and visual abrupt onsets. Journal of Experimental Psychology: Human Perception \& Performance, 20, 799-806.

TipPER, S., Driver, J., \& Weaver, B. (1991). Object-centred inhibition of return of visual attention. Quarterly Journal of Experimental Psychology, 43A, 289-298.

Tipper, S., Weaver, B., Jerreat, L. M., \& Burak, A. L. (1994). Object-based and environment-based inhibition of return of visual attention. Journal of Experimental Psychology: Human Perception \& Performance, 20, 478-499.

Treisman, A. (1988). Features and objects: The Fourteenth Bartlett Memorial Lecture. Quarterly Journal of Experimental Psychology, 40A, 201-237.

Treisman, A., \& Gelade, G. (1980). A feature integration theory of attention. Cognitive Psychology, 12, 97-136.

TsaL, Y., \& LAVIE, N. (1988). Attending to color and shape: The special role of location in selective visual processing. Perception \& Psychophysics, 44, 15-21.

Tsal, Y., \& LAVIE, N. (1993). Location dominance in attending to color and shape. Journal of Experimental Psychology: Human Perception \& Performance, 19, 131-139.

VAUGHAN, J. (1984). Saccades directed at previously attended locations in space. In A. G. Gale \& F. Johnson (Eds.), Theoretical and applied aspects of eye movement research (pp. 143-150). Amsterdam: North-Holland.

\section{NOTES}

1. Two caveats need to be made concerning our position on the deployment of attention between presentation of the cue and target stimuli. First, it is important to note that we are not assuming that a stimulus must be physically present in order for one to maintain attention to one or more of its attributes. Rather, we assume only that attention must be allocated (either implicitly or explicitly) to another value of a particular dimension (i.e., another spatial location or color) in order to constitute removal of attention from the previous value. Second, the results from one condition of Maylor and Hockey (1985, Experiment 4) raise the possibility that it may not always be necessary to remove attention from a cued location to produce inhibition of return to that location. They presented both cue and target at fixation and obtained an inhibition of return effect. If attention remained at fixation throughout 
the trial, our presumed requirement would not have been satisfied. However, without more information about the subject's attentional state during the cue-to-target interval, it is difficult to interpret this result. Nevertheless, it does leave open the possibility that our assumption about attentional removal may not apply in all situations. More work is needed in order to resolve this issue.

2 . We also performed a cue color $\times$ target color analysis of variance on the data from all three experiments. The conclusions drawn from these analyses are entirely consistent with those drawn from the analyses described in the text. In these analyses, the main effects of target color were, of course, identical to the set of analyses described in the text, there were no main effects of cue color, and cue color and target color interacted significantly for both Experiments 1 and $3(p<.0005$ and $p<.05$, respectively) indicating color inhibition of return, but not for Experiment $2(F<1)$.

\section{APPENDIX}

The possibility exists that in Experiments 1 and 3 the presentation of the cue may have raised the threshold for subjects to detect a target of the same color, owing to chromatic adaptation. If so, this difference in threshold may have produced the observed reaction time differences between repeated and nonrepeated trials. Such a possibility is counter to our assumption that the reaction time differences reflect inhibition of return from a presumably more central (possibly attentional) locus. In order to rule out this possibility, we conducted an additional experiment.

In this experiment, we manipulated the intensity of a brief red target stimulus and measured the ability of subjects to detect that target when it was preceded by either a red or a blue cue. If the cue raises the threshold to detect a target of the same color, the ability to detect the red target should be poorer when it is preceded by a red cue than when it is preceded by a blue cue.

\section{Method}

Subjects. Ten Washington University undergraduates who did not participate in any of the other experiments reported here were each paid $\$ 5$ and served in a single 30 -min session. All had normal or corrected-to-normal vision.

Stimuli. The fixation, cue, and neutral attractor stimuli as well as the background color were identical to those in Experiment 3. The target stimulus consisted of either an empty black square or a black square containing one of five different arrays of red dots. Thus, there were six different target stimuli. The five stimuli that contained dots were formed by turning red $3,9,15,21$, or 27 screen elements out of the total 357 elements that formed the black target square. We refer to these different arrays as different intensities of target stimuli. Dot positions were chosen so that coverage of the black square was of uniform density and so that no recognizable patterns were formed by the dots. The same five arrays of red dots were used throughout each session and for all subjects.

Procedure. As in Experiment 3, each trial began with the presentation of the green fixation square for $510 \mathrm{msec}$ followed by either a red or a blue cue for $900 \mathrm{msec}$ and then by the green neutral attractor for $900 \mathrm{msec}$. Then one of the six target stimuli was presented for $42 \mathrm{msec}$ immediately following the neutral attractor. The target was replaced with an empty black square, which remained until a response was made or until 4,000 msec had elapsed. Subjects responded by pressing the "/ "key if they detected red dots or by pressing the " $\mathrm{z}$ " key if they did not detect any red dots. The subjects were not provided with feedback about the accuracy of their performance and they were not told what proportion of trials would have targets with red dots. Error messages were provided if responses were made either before the target was presented or after the 4,000-msec response period had expired.

Design. Each subject was presented with eight blocks, each of which contained 36 randomly ordered trials. For each block, half the trials had a red cue and half had a blue cue. During a block, each of the six target stimuli were presented three times when the cue was red and three times when the cue was blue.

\section{Results and Discussion}

The percentage of correct responses for each target intensity is shown in Figure Al separately for trials with red and blue cues. Accuracy increased as target intensity increased $[F(5,45)=$ $54.2, p<.0001]$, and there was no effect of cue color on accuracy $[F(1,9)<1]$. There was a marginal interaction between cue color and target intensity $[F(5,45)=2.3, p<.06]$, attributable primarily to the superior performance following red cues at the 21 -dot intensity. These results show that subjects' threshold to detect the various target stimuli here was not affected by the color of the cue. We should note that it is possible that detection of the target stimuli used here may not fully capture the effect that chromatic adaptation might have had on detection of the stimuli used as targets in Experiments 1 and 3. Nevertheless, the present results suggest that the pattern obtained in Experiments 1 and 3 was not an artifact of chromatic adaptation, but instead reflects a true inhibition of return of attention to a previously attended color.

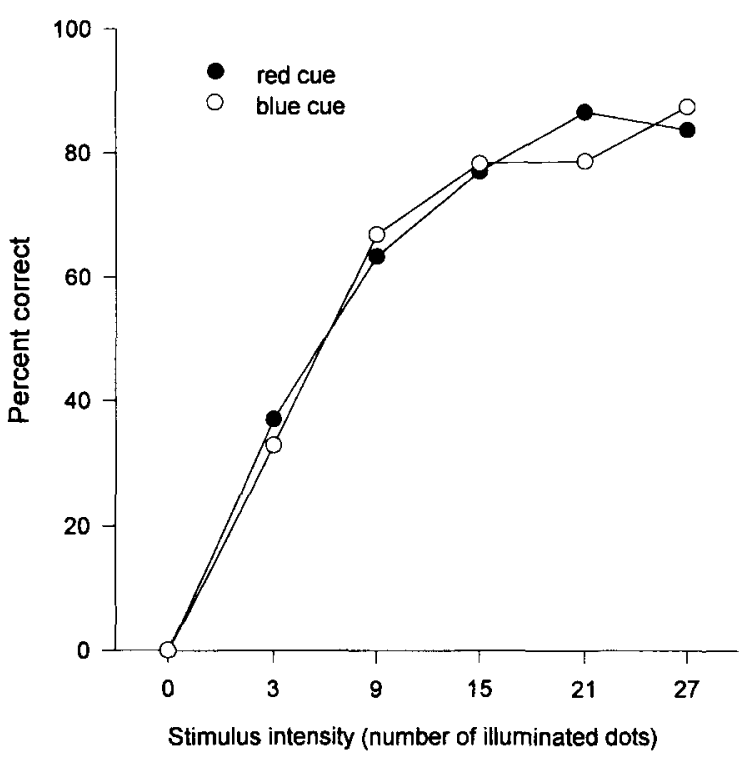

Figure A1. Percentage of correct responses as a function of target intensity and cue color.

(Manuscript received January 21, 1994; 